\title{
3D Biomechanical tongue model for management of tongue tumours: Conceptualisation to reality
}

\author{
Piyush Sinha ${ }^{1}$, Vishal Rao ${ }^{1}$, Anand Subash ${ }^{1}$, and Prasanth Puranik ${ }^{1}$ \\ ${ }^{1} \mathrm{HCG}$
}

April 29, 2020

\begin{abstract}
Background: Increasing the use of tobacco by the younger generation has increased in oral cavity tumours. Surgical treatment is radical and results in severe functional morbidity. Using computer-aided designing technology, surgical and rehabilitative planning can be better. We present here our concept of a biomechanical 3D tongue model and its clinical utility in the management of tongue tumours. Methods: Patients diagnosed with Carcinoma of Tongue were included. These patients underwent a pre-operative Magnetic Resonance Imaging (MRI) of the head and neck region at our center. These patients were informed about the use of a 3D biomechanical patient-specific model for treatment planning and execution. Using Materialise Mimics Innovation Suite 19 DICOM data was imported, visualized, edited and segmented. Flashforge 'Creator-pro'3D Printer was used for 3D Printing. Fused Deposition Modeling (FDM) technology was used to print the tumour and uninvolved tongue in two different colors for easy identification. These patients underwent surgery with the 3D model serving as a guide for margins. Results: Two patients with stage III squamous cell carcinoma of tongue underwent the surgery based on the plan evolved from the 3D model. All the surgical margins were clear. The model helped address the discordance between patient expectations and surgical outcomes. We found that the model aided the reconstructive surgeon to plan the flap volume better and this translated into better rehabilitative outcomes. Discussion and Conclusion: The 3D biomechanical tongue model is a novel concept and may aid in improving our overall treatment outcomes.
\end{abstract}

\section{D Biomechanical tongue model for management of tongue tumours: Conceptualisation to reality}

Introduction:

The tongue is one of the most commonly involved sites in the oral cavity and remains a serious health problem in many countries. The lateral border and the adjacent floor of the mouth area are the most prone to developing a malignancy ${ }^{1}$. To achieve an optimal oncological outcome, the resection margins should at least be $5 \mathrm{~mm}$ in all dimensions. Surgeons can be deceived by the two-dimensional imaging modalities to map the tumour. With the 3rd dimension often being assessed clinically and intraoperative, surgeons may often over resect or end up compromising surgical margins ${ }^{2}$. Although over resection is surgically safe, it results in the tongue losing a lot of its dynamic functions.

Modern technology has made 3D printing a reality. 3D printing is an additive manufacturing technology, which involves the generation of a Computer-Aided Design to create a 3D print by layering material one above the other. This technology is being widely used for planning bony reconstruction but has still not found much utility in soft tissue reconstruction. Having a 3D model to gauge the tumour extent can help not only the surgeon perform the ablative procedure, but also the patient and family to understand the dynamics behind the extent of resection. Discordance is often noticed between the anticipation of the patients and the final surgical outcome. A 3D assembly would help the reconstructive surgeon plan their reconstructive strategies better ${ }^{3}$. 
Rehabilitating patients following glossectomy is an uphill task. A disconnect between preoperative planning of rehabilitation and its implementation often cripples the expertise of even a trained speech-language pathologist ${ }^{4}$. A better understanding of the surgical defect by the rehabilitative team can help achieve realistic timeline based goals ${ }^{5}$.

In the present work, we focus on the design of a $3 \mathrm{D}$ patient-specific biomechanical model used by us for comprehensive treatment planning and execution.

\section{Material and Methodology:}

The study was done to evaluate the feasibility and role of a 3D patient-specific biomechanical model for the management of tongue tumour. The study was conducted at the HealthCare Global Enterprises Cancer Centre, Bangalore, India. The technical support for developing the 3D patient-specific biomechanical model was provided by Anatomiz3D LLP, Mumbai, India.

In the present study two patients, diagnosed with Carcinoma Tongue, sub-site lateral border of the tongue were included. These patients underwent a pre-operative Magnetic Resonance Imaging (MRI) of the head and neck region at our center. The images were acquired on a 3 Tesla SIEMENS Skyra ( Magnetic Resonance Imaging machine without contrast with 48 channels in multi-planar, multi-sequence of head and neck region with a pixel resolution of $0.9 \times 0.9 \times 0.9 \mathrm{~mm}^{3}$.

These patients were informed about the use of a 3D biomechanical patient-specific model for treatment planning and execution and consented for the same.

The data acquired by the MRI was obtained in a digital DICOM format. Using Materialise Mimics Innovation Suite 19 ( ), Belgium, an FDA, and CE approved software DICOM data was imported, visualized, edited and segmented. Open-source software, MakerBot MakerWare 2.4.1, Brooklyn NY USA, was used to slice the 3D model, which serves as input data for the 3D Printer (Figure 1).

Flashforge 'Creator-pro'3D Printer @ , Zhejiang, China was used. Fused Deposition Modeling (FDM) technology was used to print the tumour and uninvolved tongue in two different colors for easy identification. Recreus Filaflex (B) Elda, Spain flexible filament $1.75 \mathrm{~mm}$ spool was the print material. The filament melts because of high temperature at the nozzle and liquefied plastic is deposited on the print bed layer by layer as the previous layer cools (Figure 2).

A team of Head and Neck Surgical Oncologist, Reconstructive surgeons, Anaesthetist, Physician, and SpeechLanguage pathologist, preoperatively evaluated the patients. The 3D biomechanical patient-specific tongue model was used by the team to frame a treatment and rehabilitation plan taking into account the patient preferences and needs.

These patients underwent a partial/ hemiglossectomy with neck dissection. The 3D model served as a guide for margins. As a preoperative assessment of volume loss was now possible, the reconstructive team could simultaneously start the appropriate flap harvest. The model also enabled accurate molding of the flap into the surgical defect.

Oral swallow and speech rehabilitation were initiated from postoperative day 3 . One patient was able to take orally adequately, the nasogastric tube was removed.

\section{Results:}

The two patients had biopsy proved Squamous cell carcinoma of the lateral border of the tongue. Both patients were male. Based on clinical evaluation the patients were staged T3N0M0 (Stage III) as per the AJCC 8th Edition.

Patient 1 underwent a partial glossectomy with left selective neck dissection (I-III) and the surgical defect was reconstructed with a left radial artery forearm flap. Patient 2 underwent a hemiglossectomy with right selective neck dissection (I-III) and the defect was reconstructed with a left lateral arm flap. 
Frozen section reported the margins to be safe $(>5 \mathrm{~mm})$ in both cases. The final histopathological margin was clear in both cases (Table I).

\begin{tabular}{|c|c|c|c|c|c|c|}
\hline \multicolumn{2}{|c|}{ Patient Demographics } & Side & $\begin{array}{c}\text { Tumour Dimension on MRI } \\
(\mathrm{cms})\end{array}$ & TNM (Stage) & Closest Margin & Furthest Margin \\
\hline Patient 1 & Male & Left & $2.2 \times 1.1 \times 1.6 \mathrm{~cm}$ & $\mathrm{~T}_{3} \mathrm{~N}_{0} \mathrm{M}_{0}(\mathrm{III})$ & 7 & 10 \\
\hline Patient 2 & Male & Right & $2.1 \times 2.8 \times 2.2 \mathrm{~cm}$ & $\mathrm{~T}_{3} \mathrm{~N}_{0} \mathrm{M}_{0}$ (III) & 6 & 9 \\
\hline
\end{tabular}

Table 1: Patient Demographics

\section{Discussion:}

The tongue is a dynamic organ and has often tricked the surgeon. A close or positive margin has adverse implications on treatment outcome and overall survival ${ }^{6}$. With better imaging modalities the surgeon can better assess the tumour extent. However, a real-time assessment of tumour intra-operatively is far from reality. The decision as to how much to resect is taken based on clinical judgment. Over resection and margin positivity in the third dimension is always a threat ${ }^{7}$. The biomechanical 3D model used by us helps the surgeon to get a real-time three-dimensional extent of the tumour. In our study, we noted that resection was more accurate and resulted in lesser chances of over resection.

Until recently, reconstructive surgeons designed free flaps by visually estimating the defect and using basic geometric shapes. However, defects following ablative procedures are considerably more complex. Today CAD-CAM 3D printed models have increased the accuracy of reconstruction in the management of buccoalveolar complex tumours. A similar guide for tongue tumour resection can help improve the efficacy of reconstruction ${ }^{8}$. Owing to the dynamic nature of the tongue, the extent of functional restriction often relates to the extent of resection ${ }^{9}$. With a realtime model, the patient and family can better visualize the tumour and understand the concept of safe margin and possible extent of resection. This helps them better prepare for the outcomes and functional deficits. With a wealth of information available at a click of a button, the patients also prefer to be fully prepared for the deficits to make an appropriate decision.

Due to incessant tobacco use amongst youngsters, the disease is now more prevalent in the younger population. Functionally rehabilitating these young patients accurately has definitive implications on quality of life personally and professionally. Often the resected specimen is used as a benchmark to plan the flap volume. A common problem encountered by patients post-reconstruction is often due to preventable causes. The flap bulk can restrict tongue movements, impair swallowing and compromise of vocalization ${ }^{10}$. The extra flap bulk which is recommended can be optimized with the 3D model reducing the chances of such problems.

Using the 3D model the rehabilitative team also can have a better understanding of the resection and functional restriction. This helps in developing better rehabilitative strategies ${ }^{9}$.

\section{Conclusion:}

Though the current technology is not yet advanced enough to create a dynamic model to exactly mimic the tongue and many would argue against its utility. Nevertheless, the 3D biomechanical model might help bridge the existing deficiencies in our treatment to deliver the best outcomes.

\section{References}

1) Moore S, Johnson N, Pierce A, Wilson D.The epidemiology of tongue cancer: a review of global incidence. Oral Diseases, (2008) 6(2), 75-84. doi:10.1111/j.1601-0825.2000.tb00105.x

2) Idris S, Mlynarek AM, Ansari K. et al. Multi-dimensional analysis of oral cavity and oropharyngeal defects following cancer extirpation surgery, a cadaveric study. J of Otolaryngol - Head \& Neck Surg (2018) 47: 27. https://doi.org/10.1186/s40463-018-0276-9 
3) Shilo D, Emodi O, Blanc O, Noy D, Rachmiel A. Printing the Future-Updates in 3D Printing for Surgical Applications. Rambam Maimonides Med J. 2018;9(3):e0020.doi:10.5041/RMMJ.10343

4) Bachher GK, Dholam K, Pai PS. Effective rehabilitation after partial glossectomy. Indian J Otolaryngol Head Neck Surg. 2002;54(1):39-43. doi:10.1007/BF02911004

5) H Schliephake, R Schmelzeisen, R Schönweiler, T Schneller, and C Altenbernd,. Speech, deg- lutition and life quality after intraoral tumour resection. A prospective study; International Journal of Oral and Maxillofacial Surgery. 1998;27(2):99-105

6) Loree TR, Strong EW.Significance of positive margins in oral cavity squamous carcinoma. The American Journal of Surgery.1990;160(4), 410-4. doi:10.1016/s0002-9610(05)80555-0

7) Shun Miyota, Takanori Kobayashi, Tatsuya Abé, et al., "Intraoperative Assessment of Surgical Margins of Oral Squamous Cell Carcinoma Using Frozen Sections: A Practical Clinicopathological Management for Recurrences," BioMed Research International, vol. 2014, Article ID 823968, 9 pages, 2014. https://doi.org/10.1155/2014/823968.

8) Antony A K, Chen W F, Kolokythas A, Weimer K A, Cohen MN. Use of virtual surgery and stereolithographyguided osteotomy for mandibular reconstruction with the free fibula. Plastic and Reconstructive Surgery. 2011; 128(5), 1080-4. https://doi.org/10.1097/PRS.0b013e31822b6723

9) S Buchaillard, M Brix, P Perrier, Y Payan. Simulations of the consequences of tongue surgery on tongue mobility: implications for speech production in post-surgery conditions. The International Journal of Medical Robotics and Computer Assisted Surgery.2007;3(3) 252- 61.

10) Kimata Y, Sakuraba M, Hishinuma S, et al. Analysis of the relations between the shape of the reconstructed tongue and postoperative functions after subtotal or total glossectomy. The Laryngoscope.2003; 113(5), 905-9. https://doi.org/10.1097/00005537-200305000-00024

Legends

Figure 1: MRI Images post processing

Figure 2: 3D reconstituted tongue

Figure 3: 3D printed biomechanical tongue model

Table 1 : Patient clinical details
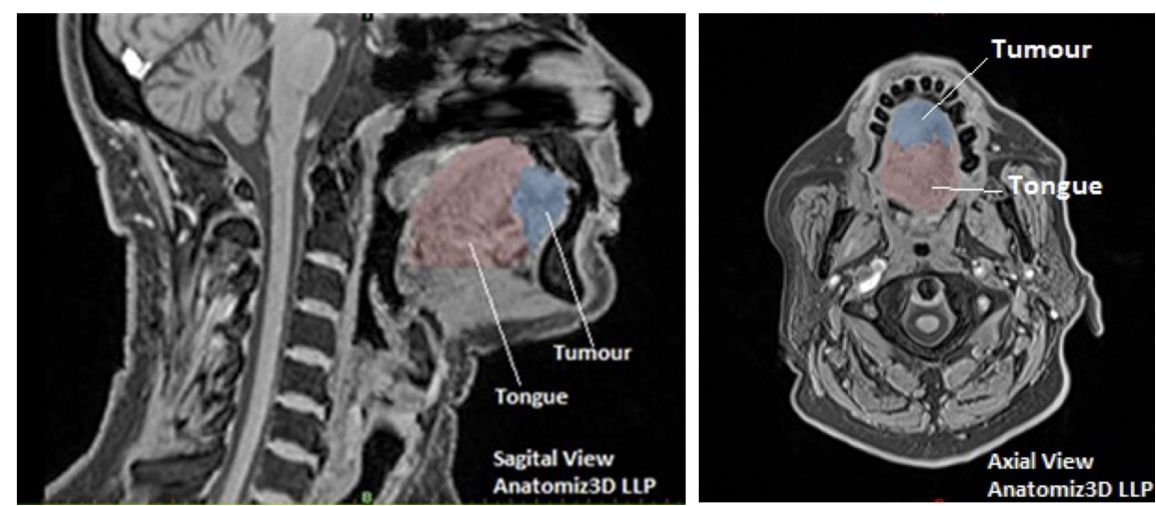

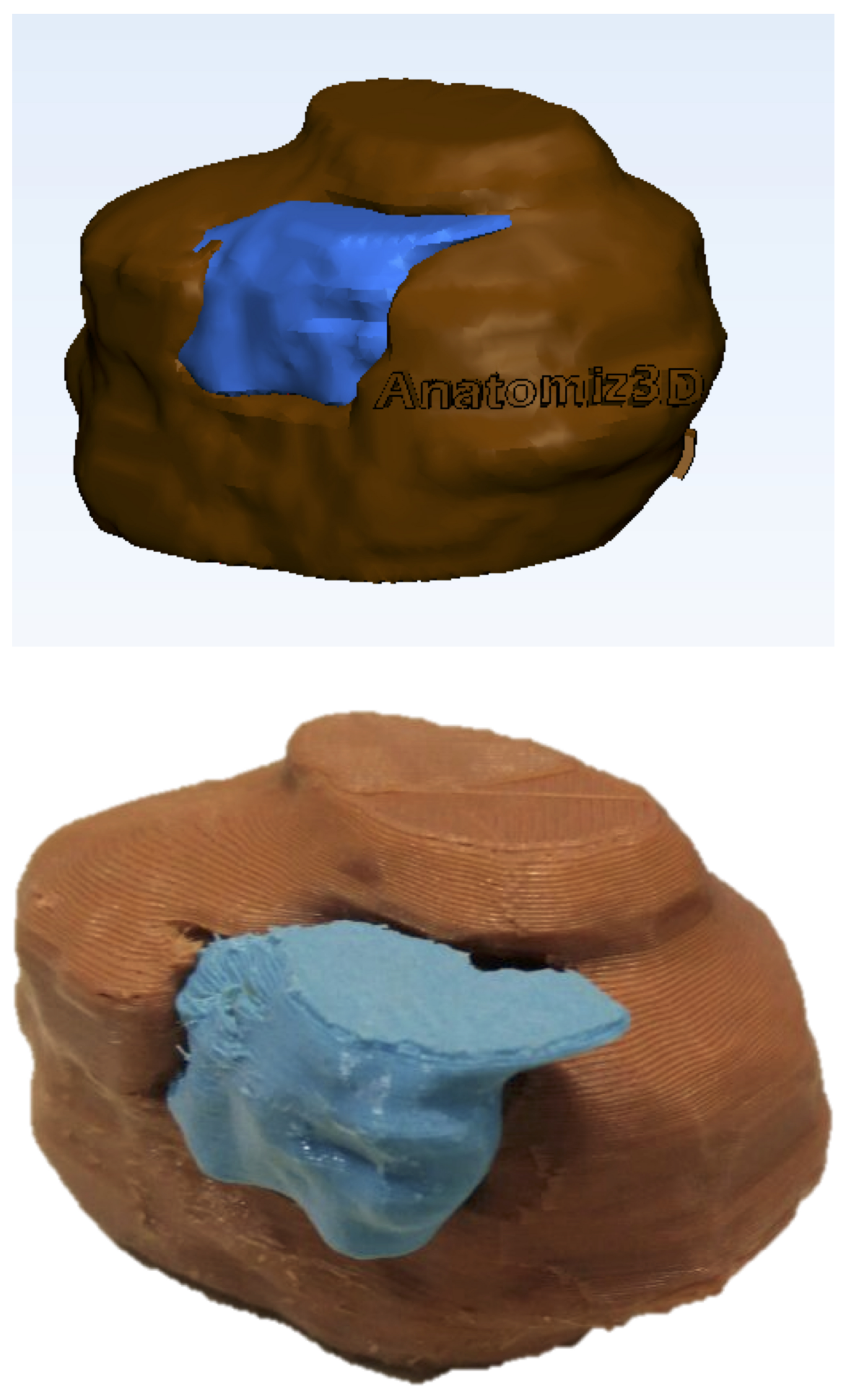